\title{
Étude expérimentale de l'émission $X$ d'agrégats nanométriques d'argon irradiés par une impulsion laser intense
}

\author{
T. Caillaud, F. Blasco, F. Dorchies, Y. Glinec, C. Stenz et J. Stevefelt \\ Centre Lasers Intenses et Applications, UMR 5107 du CNRS, Université de Bordeaux l, France
}

\begin{abstract}
Résumé. L'émission X de la couche $\mathrm{K}$ des agrégats de l'argon irradiés par des impulsions laser de $10^{17} \mathrm{~W} \mathrm{~cm}^{-2}$ était résolue spectralement et examinée en fonction de différents paramètres laser et agrégats. Un effort particulier était d'abord consacré au contrôle de la focalisation du laser dans le jet d'agrégats, et du contraste temporel de l'impulsion laser, comme ces deux paramètres étaient observés d'avoir une influence drastique sur l'émission X. Le rayon moyen des agrégats était ensuite varié de 200 à $350^{\circ}$, et aussi la durée des impulsions laser de $30 \mathrm{fs}$ à 2 ps (largeur à mi-hauteur). L'émission X était observée d'atteindre une valeur maximale pour une certaine durée de l'impulsion laser (autour de quelques $100 \mathrm{fs}$ ), qui augmentait avec le rayon moyen des agrégats. Ces observations pouvaient être comprises en termes d'un effet de résonance en fonction de la densité électronique. La durée de l'émission X était aussi examinée audessus de $3 \mathrm{keV}$ avec un caméra à balayage (résolution d'environ 1 ps à $1 \mathrm{kHz}$ ). Cette durée était trouvée d'être environ 2 ps (largeur à mi-hauteur) pour les conditions optimales d'émission X.
\end{abstract}

\section{INTRODUCTION}

De nombreux travaux de recherche [1-3] ont été menés depuis quelques années sur l'interaction d'une impulsion laser intense avec des agrégats nanométriques. Ces agrégats d'atomes peuvent être obtenus par condensation spontanée d'un gaz rare lors de sa détente dans une buse supersonique. Ces agrégats présentent localement une densité proche de celle du solide susceptible d'induire une absorption collisionnelle plus élevée qu'avec une cible gazeuse. D'autre part, la quantité de matière chauffée par l'impulsion laser étant confinée aux seuls agrégats, les débris produits après l'interaction sont moins nombreux qu'avec une cible solide. Plusieurs expériences ont ainsi montré le potentiel de cette cible pour la production de particules énergétiques (UV, X, ions, neutrons, ...) à haute cadence et sans débris [4,5]. Plus récemment, des études ont été menées sur la dynamique temporelle dans l'interaction laser-agrégats $[6,7]$.

La géométrie nouvelle de cette cible agrégat dont la taille caractéristique est très petite devant la longueur d'onde laser donne lieu à de nouvelles questions quant aux mécanismes physiques impliqués. En effet, plusieurs observations expérimentales ne sont pas encore expliquées, à commencer par des états de charge ioniques excessivement élevés.

L'objectif de notre étude est double. Il s'agit, d'une part, de comprendre les mécanismes gouvernant cette interaction via l'émission $\mathrm{X}$ qui en résulte. On attend de ce rayonnement $\mathrm{X}$ qu'il soit émis dans les premières picosecondes qui suivent l'interaction, pendant que le nano-plasma créé est encore suffisamment dense et chaud. Le spectre de ce rayonnement est caractéristique des états de charge présents dans le plasma émetteur. Son étude, éventuellement résolue en temps, est par conséquent une source d'information particulièrement riche sur la dynamique initiale de l'agrégat irradié. D'autre part, nous nous proposons de développer et d'optimiser une source de rayonnement $\mathrm{X}$ de haute cadence et sans débris, basée sur ce schéma d'interaction.

Dans cet article, nous présentons une étude du rayonnement $\mathrm{X}$ émis par des agrégats d'Argon irradiés par une impulsion laser de $10^{17} \mathrm{~W} . \mathrm{cm}^{-2}$ d'intensité maximale et de $30 \mathrm{fs}$ de durée minimale à mi-hauteur. Ce rayonnement a été résolu spectralement dans la gamme de longueur d'onde correspondant à l'émission de couche $\mathrm{K}$ de l'Argon (autour de $3 \mathrm{keV}$ ). La taille de la source, ainsi que le nombre absolu de photons X ont aussi été déterminés. Dans un premier temps, un effort a été réalisé en vue de contrôler le contraste temporel de l'impulsion laser à l'échelle de la nanoseconde ainsi que le plan de focalisation du laser dans le jet d'agrégats: deux paramètres dont l'importance s'est avérée déterminante pour l'intensité de l'émission $\mathrm{X}$. Par la suite, le rayonnement $\mathrm{X}$ a été étudié en fonction de la taille des agrégats et de la durée de 
l'impulsion laser à énergie constante. Enfin, nous avons étudié l'aspect temporel de ce rayonnement dans les conditions optimales d'émission X.

\section{ETUDES PRELIMINAIRES}

\subsection{Caractérisation du laser et de la cible}

La chaîne laser du CELIA délivre des impulsions avec une cadence de $1 \mathrm{kHz}$. En sortie de chaîne, le faisceau est séparé en deux bras, dont un a été utilisé lors des expériences présentées dans cet article. Sur ce bras, chacune des impulsions atteignait $4 \mathrm{~mJ}$ et 30 fs après compression. Elle était par la suite focalisée dans une enceinte sous vide, à l'aide d'un miroir parabolique hors axe. La quantité d'énergie laser contenue dans la tache focale ( $3 \mu \mathrm{m}$ de rayon en 1/e de l'intensité maximale) et dans les 30 fs de durée à mi-hauteur a été mesurée autour de $1 \mathrm{~mJ}$. Cette mesure conduit à une intensité maximale de $10^{17} \mathrm{~W} . \mathrm{cm}^{-2}$ au foyer de la parabole.

Afin d'obtenir le plus grand nombre possible de photons $\mathrm{X}$, nous avons focalisé l'impulsion laser dans le jet de gaz directement à la sortie de la buse, où le nombre d'agrégats attendus est plus important. Le mélange de gaz et d'agrégats qui constituent la cible de l'impulsion laser a été caractérisé. Ce travail est basé sur deux diagnostics optiques et supporté par un code de simulation numérique [8]. Les résultats présentés ici ont été obtenus avec une buse conique. A sa sortie, le profil de la densité atomique totale $n_{t o t}$ est un créneau de $4 \mathrm{~mm}$ de diamètre.

La densité homogène dans le jet suit la loi: $n_{t o t}\left(\mathrm{~cm}^{-3}\right)=1,25 \cdot 10^{17} P_{i n}$ (bars), où $P_{i n}$ est la pression d'argon en amont de la buse. Elle est contrôlée par un manomètre. Environ $75 \%$ de ces atomes sont dans une phase gazeuse. Les autres sont regroupés dans des agrégats dont la répartition est homogène dans le jet. Leur rayon moyen peut être ajusté entre 200 et $350 \AA$ A, en variant la pression $P_{\text {in }}$ depuis 20 jusqu'à 60 bars.

\subsection{Détermination du plan focal optimal}

Une partie importante du jet est sous forme de gaz dont l'ionisation peut induire une réfraction violente du faisceau. Ce mécanisme a pour effet de diminuer l'intensité de l'impulsion laser en son foyer [9]. L'émission X a été mesurée en fonction de la position du plan de focalisation dans le jet. Lors de ces mesures, il a été observé un nombre total de photons $X$ émis atteignant une valeur maximale pour des profondeurs de 100 à $500 \mu \mathrm{m}$ à l'intérieur du jet en fonction de la pression $P_{i n}$ de travail (respectivement égale à 60 et 20 bars). Une focalisation plus en avant dans le jet s'accompagnait d'une décroissance du nombre d'X jusqu'au seuil de détection après environ $1 \mathrm{~mm}$. La profondeur optimale du plan de focalisation a été déterminée par ces mesures. Aucune modification significative de cette grandeur n'a été observée en faisant l'expérience avec des durées laser plus longues ( $300 \mathrm{fs}$ et $1 \mathrm{ps}$ ) et à énergie constante.

L'ensemble des mesures présentées dans la suite de cet article ont été réalisées au plan de focalisation

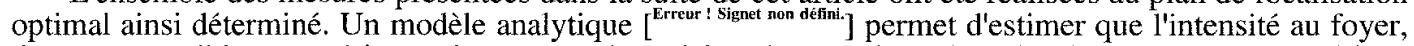
dans ces conditions expérimentales, reste très voisine de sa valeur dans le vide. Dans ces conditions expéri-mentales maîtrisées, l'intensité laser dans le volume focal était donc de l'ordre de $10^{17} \mathrm{~W} . \mathrm{cm}^{-2}$ (à 30 fs) et la densité d'agrégats était déterminée par les profils mesurés (Cf. article "Caractérisation de jets d'agrégats d'Argon pour l'étude de l'interaction laser - agrégats").

\section{MESURES DE L'EMISSION $X$}

\subsection{Dispositif expérimental}

Plusieurs diagnostics $\mathrm{X}$ ont été mis en place dans l'enceinte d'interaction sous vide:

- Un spectromètre $X$ de haute résolution spectrale et imageur dans une dimension spatiale. Il analysait le rayonnement $X$ à $90^{\circ}$ du faisceau laser. La dimension spatiale le long de l'axe du faisceau laser était résolue. Ce spectromètre est constitué d'un cristal sphérique de Mica et d'une caméra CCD X. La géométrie et les caractéristiques détaillées de ce spectromètre sont décrits dans un article [10].

- Une caméra streak ultra rapide (résolution de 1.2 ps au $1 \mathrm{kHz}$ et 1 million de tirs) observant la durée du rayonnement $\mathrm{X}$ émis autour de $3 \mathrm{keV}$.

Des aimants étaient placés devant chacun de ces diagnostics, de façon à s'affranchir de la contribution éventuelle de particules chargées aux signaux recueillis. 


\subsection{Influence du contraste temporel à l'échelle ns}

L'architecture de la chaîne laser donne lieu à une pré-impulsion précédant de 4 ns l'impulsion laser principale. Le contraste $C_{4 n s}$ défini comme le rapport des intensités de ces deux impulsions peut être modulé de 10 jusqu'à $10^{6}$ par une cellule de Pockels.

Nous avons observé que le nombre de photons $X$ détectés ne dépendait pas de la valeur de ce contraste jusqu'à une valeur critique. Au-delà de cette valeur (de l'ordre de $10^{3}$ à $30 \mathrm{fs}$ et de $10^{2}$ à $300 \mathrm{fs}$ ), le nombre de photons $\mathrm{X}$ décroissait pour atteindre un niveau proche du seuil de détection avec $C=10$. A la valeur critique du contraste, l'intensité maximale de la pré-impulsion était de l'ordre de $10^{14^{4 n g}} \mathrm{~W} \cdot \mathrm{cm}^{-2}$, c'est-à-dire de l'ordre de l'intensité seuil pour que la pré-impulsion puisse arracher un premier électron à un atome isolé d'argon.

Ces données semblent indiquer que la perturbation induite par une impulsion laser sur un agrégat d'argon ne commence qu'avec l'ionisation d'un de ses atomes dans le champ électrique du laser. Une fois ainsi perturbé, sa durée de vie sous forme agrégée semble inférieure à 4 ns. Les résultats présentés dans la suite de cet article ont été obtenus avec le meilleur contraste $C_{A n s}=10^{6}$, plusieurs ordres de grandeurs sous sa valeur critique. Ceci garantit l'interaction de l'impulsion principale avec des agrégats non perturbés.

\subsection{Spectre de l'émission $X$}

Des spectres $\mathrm{X}$ ont été enregistrés dans les conditions de focalisation et de contraste précédemment indiquées. Cette étude a été réalisée en fonction de différentes valeurs de la durée de l'impulsion laser $\Delta t_{t a s}$ et du rayon $R$ des agrégats. La durée $\Delta t_{\text {las }}$ était modifiée avec la distance entre les réseaux du compresseur et contrôlée par un auto-corrélateur du deuxième ordre. Le rayon $R$ était ajusté par la pression en amont de la buse.

Un spectre $\mathrm{X}$ obtenu avec $R=350 \AA$ et $\Delta t_{t a s}=500 \mathrm{fs}$ FWHM est représenté sur la figure 1a. Il est schématiquement constitué de la raie de résonance $\mathrm{He}_{\alpha}$ et de ses satellites. La forme générale des spectres ęst assez indépendante des deux paramètres $\Delta t_{\text {las }}$ et $R$, exceptée pour le cas des plus gros agrégats $(R=350$ A) irradiés avec les impulsions les plus brèves ( $30 \mathrm{fs}$ ). Dans ce cas, la contribution relative des satellites devient plus importante (Cf. Fig. 1b).

La présence de la raie de résonance $\mathrm{He}_{\alpha}$ démontre la création d'ions $\mathrm{Ar}^{16+}$ lors de l'interaction alors que le seuil d'ionisation des électrons en couche $\mathrm{K}$ pour un atome seul dans le champ laser se situe autour de $10^{19} \mathrm{~W} . \mathrm{cm}^{-2}$. C'est le degré d'ionisation le plus élevé que nous ayons observé: aucune émission dans la raie $\mathrm{H}_{\alpha}\left(\mathrm{Ar}^{17 t}\right)$ n'a été détectée.

\subsection{Comportement avec la durée de l'impulsion laser}

La figure 2 représente l'intensité $\mathrm{X}$ mesurée dans la raie de résonance $\mathrm{He}_{\alpha}$ en fonction de la durée laser. L'énergie laser était constante lors de cette expérience: l'augmentation de la durée de 30 fs à $300 \mathrm{fs}$ s'accompagnait d'une décroissance de l'intensité maximale depuis $10^{17}$ jusqu'à $10^{16} \mathrm{~W} . \mathrm{cm}^{-2}$. Le rayon moyen des agrégats était de $300 \AA$ A. Une durée optimale a clairement été mise en évidence. Elle est de l'ordre de $500 \mathrm{fs}$ FWHM avec ces agrégats les plus gros. L'expérience a été réalisée avec des agrégats de taille moyenne variable. Un phénomène identique a été observé. La durée laser optimale croît avec le rayon moyen des agrégats.

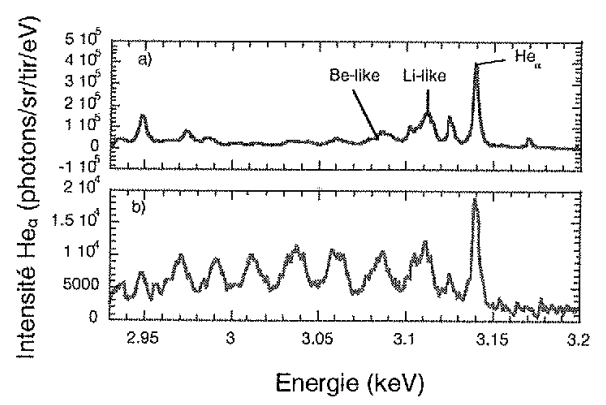

Figure I: Spectres $X$ de couche $K$ émis par des agrégats d'Argon de $300 \AA$ de rayon irradiés par une impulsion laser de durée $\Delta t_{\text {ta." }}$ a) $\Delta t_{i a s}=500 \mathrm{fs}$ FWHM. b) $\Delta t_{\text {tas }}=30 \mathrm{fs}$ FWHM.

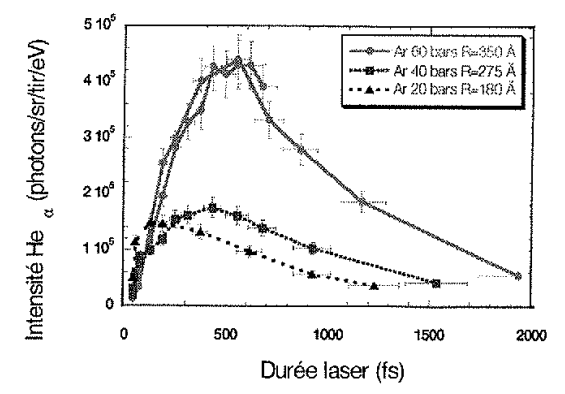

Figure 2: Intensité de l'émission $X$ des agrégats dans la raie $H e_{\alpha}$ de l'Argon en fonction de la durée de l'impulsion laser. Le rayon moyen des agrégats varie de 180 à $350 \AA$. 


\subsection{Durée d'émission $X$}

Nous avons également étudié la durée de l'émission $\mathrm{X}$ autour de $3 \mathrm{keV}$ au moyen d'une caméra streak. Pour ces mesures, nous nous sommes placé dans les conditions optimales de l'émission X. Le signal recueillit est intégré sur 36000 tirs laser pour obtenir un rapport signal/bruit suffisant pour observer la trace de l'émission X. Les résultats obtenus sont présentés dans la figure 3. La durée de l'émission X pour des agrégats d'argon à 60 bars $(\cong 350 \AA)$, en tenant comptes des différentes contributions d'élargissement temporel liées au dispositif expérimental, est d'environ ( $2.2 \pm 0.8)$ ps. Cette durée comprends l'extension spatiale du plasma et donc, le temps de parcours de l'onde qui pour un plasma de $300 \mu \mathrm{m}$ est d'environ 1 ps.

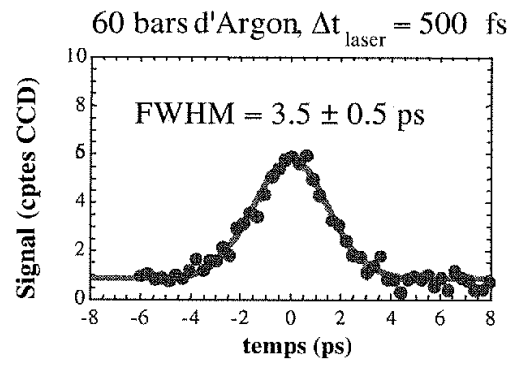

Figure 3 : Trace balayée en temps de l'émission $\mathrm{X}$ autour de $3 \mathrm{keV}$. Vitesse de balayage $\bullet 23 \mathrm{ps} / \mathrm{mm}$ Contribution statique : 1.7 ps.

Contribution géométrique : 1 ps.

Contribution photocathode : $500 \mathrm{fs}$.

Contribution gigue : $1.4 \mathrm{ps}$.

Durée d'émission : $2.2 \pm 0.8$ ps FWHM

\section{DISCUSSION}

La connaissance théorique de la réflectivité du cristal de Mica ainsi que la géométrie complète du spectromètre $X$ permet d'estimer le nombre absolu de photons $X$ émis. Cette estimation a été effectuée en considérant une émission isotrope dans $4 \pi$ stéradians et en intégrant le spectre de la couche $\mathrm{K}$ (autour de $3 \mathrm{keV}$ ). La calibration de ce spectromètre a été confirmée avec un détecteur absolu au CEA Saclay. Dans les conditions optimales $\left(R=350 \AA\right.$ et $\Delta t_{l a s}=500 \mathrm{fs}$ FWHM $)$, l'émission X ainsi estimée était de l'ordre de $10^{8}$ photons par tir laser. Connaissant le volume émetteur et la concentration des agrégats dans ce volume, on en déduit l'émission d'un seul agrégat. Elle est de 5\% photon de couche $\mathrm{K}$ par atome agrégé.

Un travail de simulation basé sur les mécanismes physiques proposés par T. Ditmire [11] est en cours pour reproduire ces résultats. Beaucoup de choses ne sont pas encore expliquées, à commencer par l'obtention d'ions $\mathrm{Ar}^{10+}$. Les résultats en fonction de la durée laser semblent indiquer qu'il faille attendre que l'agrégat s'étende jusqu'à une certaine densité pour que le processus d'absorption soit efficace. Le même type de phénomène a été observé lors de mesures d'absorption par J. S. Zweiback [12]. Ces derniers résultats sont interprétés comme l'effet de la résonance de Mie apparaissant à 3 fois la densité critique pour un nano-plasma de taille inférieure devant la longueur d'onde.

\section{CONCLUSION}

Nous avons mesuré le rayonnement $\mathrm{X}$ de couche $\mathrm{K}$ d'agrégats d'argon irradiés par une impulsion laser. L'intensité de ce rayonnement est très sensible aux conditions expérimentales: contraste temporel de l'impulsion, plan de focalisation dans le jet, une durée laser optimale (700 fs) pour un rayon d'agrégat donné $(300 \AA)$. Ils sont en partie émis par des ions très chargés $\left(\mathrm{Ar}^{16+}\right)$ dont la présence lors de l'interaction n'est pas encore totalement comprise.

\section{Remerciements}

Ce travail a été en partie financé par le Fond Européen de Développement Economique Régional, le Conseil Régional d'Aquitaine et la Russian Foundation for Basic Research (projet n ${ }^{\circ}$ 00-01-00562).

\section{Références}

[1] T. Ditmire et al., Phys. Rev. A 57 (1998) 369

[2] C. Rose-Petruck et al., Phys. Rev. A 55 (1997) 1182

[3] Mc. Pherson et al., Appl. Phys. B 57 (1993) 337

[4] Y. Shao et al., Phys. Rev. Lett. 77 (1996) 3343

[5] T. Ditmire et al., Nature 398 (1999) 489

[6] J. S. Zweiback et al., Phys. Rev, A 59 (1999) R3166
[7] L. Köller et al., Phys. Rev. Lett. 82 (1999) 3783

[8] A.C. Boldarev et al. JETP Letters, 73 ((2001) 514

[9] T. Auguste et al., Opt. Comm. 89 (1992) 145

[10] F. Blasco et al., Rev. Sci. Instr. 72 (2001) 1956

[11] T. Ditmire et al., Phys. Rev. A 53 (1996) 3379

[12] J.S. Zweiback et al., Optics Express 6 (2000) 36 